\title{
Top-down fabrication of very-high density vertically stacked silicon nanowire arrays with low temperature budget
}

\author{
Michael Zervas*, Davide Sacchetto, Giovanni De Micheli, Yusuf Leblebici \\ EPFL - Ecole Polytechnique Fédérale de Lausanne, 1015, Switzerland
}

\section{A R T I C L E I N F O}

\section{Article history:}

Received 28 January 2011

Received in revised form 26 April 2011

Accepted 20 June 2011

Available online 26 June 2011

\section{Keywords:}

TMAP

DRIE

Nanowire

Stacked

3D

Array

\begin{abstract}
A B S T R A C T
We report on a top-down complementary metal oxide semiconductor (CMOS) compatible fabrication method of ultra-high density Si nanowire (SiNW) arrays using a time multiplexed alternating process (TMAP) with low temperature budget. The flexibility of the fabrication methodology is demonstrated for curved and straight SiNW arrays with different shapes and levels. Ultra-high density SiNW arrays with round or rhombic cross-sections diameters as low as $10 \mathrm{~nm}$ are demonstrated for vertical and horizontal spacing of $60 \mathrm{~nm}$. The uniqueness of the technique, which achieves several advantages such as bulk-Si processing, low-thermal budget, and wide process window makes this fabrication method suitable for a very broad range of applications such as nano-electro-mechanical systems (NEMS), nano-electronics and bio-sensing.
\end{abstract}

(ㄷ) 2011 Elsevier B.V. All rights reserved.

\section{Introduction}

Recently, there has been a huge interest in the physical properties of Si nanowires (SiNW) due to their excellent mechanical [1] and electrical properties [2]. The one dimensionality of SiNWs makes these structures excellent candidates for sensing [3] as well as channels for solid-state field effect transistor (FET) devices [4]. Thus, reliable and inexpensive top-down fabrication methods of SiNWs are of huge interest for several applications. In this respect, stacking multiple layers of nanowires is emerging as potentially powerful approach for building high density systems [5].

One method, proposed by Javey et al. [6], relies on the iteration of contact printing and metallization techniques to stack several layers of nanowire-based circuits. The key advantage of the technique is that it can be applied to any type of nanowires grown by bottom-up approaches and it can be applied to any kind of substrates, including flexible plastics. However, the throughput of the method is limited by the number of steps and the use of different substrates for nanowire growth and pattern transfer. Another method to produce vertically-stacked SiNWs is based on Si/SiGe epitaxy and has been reported by several groups [7-9]. The epitaxial method allows for very tight control of the film thickness and therefore, of nanowire dimensions; however, it lacks fabrication compatibility with conventional CMOS processes. Indeed, the number of stacked SiNW levels on a wafer is fixed by the epitaxial

\footnotetext{
* Corresponding author.

E-mail address: michael.zervas@epfl.ch (M. Zervas).
}

layers and careful control on the stress-build up is required in order to obtain reproducible electrical characteristics [9]. A third method recently published $[10,11]$ leverage on the formation of $\mathrm{SiO}_{2}-\mathrm{Si}_{3} \mathrm{~N}_{4}$ dielectric cavities in which ultradense polysilicon nanowires can be conformally grown and etched. In particular, in [11] low horizontal pitch of about $150 \mathrm{~nm}$ and a vertical pitch of $50 \mathrm{~nm}$ are demonstrated. Another method uses time multiplexed alternating process (TMAP) etching, also known as deep reactive ion etching (DRIE) or Bosch process in combination with high temperature oxidation to form vertical stacks of SiNWs. Several groups, including the authors, have reported on variations of the TMAPDRIE process for the fabrication of vertically stacked SiNWs arrays for micro-electro-mechanical-systems MEMS [12] and FET devices $[13,14]$. Nevertheless, the SiNW fabrication typically relies on thermal oxidation and/or hydrogen annealing techniques [7,8,13,14] which increase the temperature budget.

This work focuses on the top-down fabrication of ultra-high density vertically-stacked SiNWs arrays with sub-50 nm diameter from a single etching step with low thermal budget, without the need for thermal oxidation or hydrogen annealing techniques. For instance, since all processing steps are performed at room temperature,this feature can be used in CMOS post-processing or for flexible polymeric substrates [15], for which temperatures lower than $200{ }^{\circ} \mathrm{C}$ are needed. Very-high horizontal and vertical SiNWs density is demonstrated for curved and straight geometries with edge-to-edge SiNW spacings down to $60 \mathrm{~nm}$ for a cross-sectional density of $2 \times 10^{10} / \mathrm{cm}^{2}$. The etching recipes are optimized to obtain either round- or rhombic-shaped SiNWs. Si fins are also fabricated. 
Additionally, the number of vertically placed SiNWs in-a-stack can be easily tuned from 1 to 6 .

In Section 2 the typical fabrication steps are summarized. Section 3 details the TMAP physics of etching at the nano-scale and the process developments required for optimization of the different vertically-stacked SiNW arrays designs. In Section 4 we discuss on the obtained fabricated structures. Finally, in Section 5 we draw the conclusions.

\section{Device fabrication}

The process starts with a bulk-Si wafer. The wafer is dipped for $60 \mathrm{~s}$ in tetramethylammonium hydroxide (TMAH) for priming the Si surface for hydrogen silsesquioxane (HSQ) coating, after it is dehydrated on a hot plate at $180^{\circ} \mathrm{C}$ for $10 \mathrm{~min}$. A $150 \mathrm{~nm}$ thick HSQ layer is spin coated at $4000 \mathrm{rpm}$. Then single lines (Fig. 1a) and dense arrays (Fig. 1b) are patterned using Vistec EBPG5000 e-beam lithography system. Typical HSQ lines are written with $50 \mathrm{~nm}$ width with line distance from $50 \mathrm{~nm}$ to $500 \mathrm{~nm}$. After exposure the wafer is developed in TMAH and rinsed in water, acetone and IPA, in order to lower surface tension and avoid stiction. After the exposure the wafers are processed in Alcatel AMS 200 DSE Fluorine etcher using different TMAP recipes. Four process parameters are tuned; flow rate, step time of $\mathrm{SF}_{6}$ and $\mathrm{C}_{4} \mathrm{~F}_{8}$ gases and one design parameter, the distance of the nanowire to the sidewall and pitch for the arrays. In particular, round (see Fig. 1c and d) and rhombic (see Fig. 1e and f) cross-sections can be obtained using optimized recipes, as will be explained in Section 4.

\section{Nano-scale etching}

The presented work is based on time multiplexed alternating process (TMAP) [16], also known as DRIE or Bosch process. The more advanced etching recipes presented in this work target

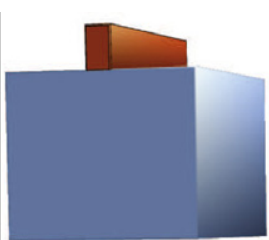

a

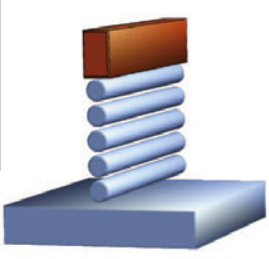

C

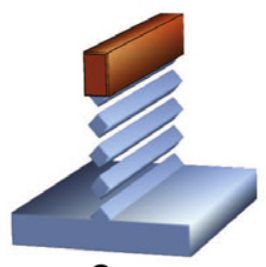

e

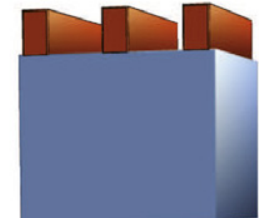

b

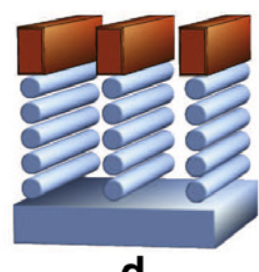

d

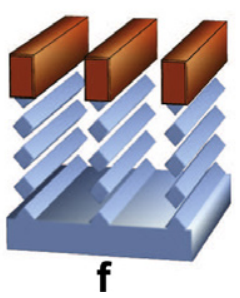

Fig. 1. Fabrication flow. (a) Single HSQ line patterned on Si substrate. Typical HSQ width is $50 \mathrm{~nm}$. (b) Dense HSQ line arrays. (c and d) After etching with recipe (2) as reported in Table 3. (e and f) After etching with recipe (3) as reported in Table 3. scallops dimension between 20 and $30 \mathrm{~nm}$, to allow the formation of stacks of nanowires from mask line widths of $50 \mathrm{~nm}$ and below. The etching rate and profile are mainly determined by three key phenomena:

- Loading effect [17].

- Microloading effect $[17,18]$.

- Ion induced defluorination of the passivation film (IIDPF) $[19,20]$.

A quick summary of design and process parameters dependencies with these physical phenomena is given in Table 1 . More details are explained in the following paragraphs. The loading effect [17] is the decrease of the etching rate of the reactive ion etching due to the increased area exposed to the $\mathrm{SF}_{6}$ plasma. In our test structures more than $90 \%$ of total wafer area is exposed. In case the pattern is required to cover more than $50 \%$ of the total wafer area then the loading effect cannot be used as part of the strategy to lower the etch rate. A special case of the loading is the microloading effect. The microloading effect is the etching rate degradation due to the high density of a pattern, the main mechanism for microloading is, etchant depletion $[17,18]$. Etchant depletion happens inside an etching trench and it is due to the difficulty for the etchant species to be replenished, as the etching opening reducing. The smaller the nanowire pitch, the lower the etch rate. Microloading can be controlled by flow rate. Thus, reducing the flow rate it reduces the rate at which the etchants are replenished leading to etchant depletion, magnifying the microloading effect. The TMAP consists of the alternation of etching and passivation steps. At the beginning of each etching cycle the plasma needs to remove the passivation at the bottom, deposited at the passivation cycle. The time needed for the passivation removal is a function of its thickness, which in turns is controlled by $\mathrm{C}_{4} \mathrm{~F}_{8}$ flow rate and time step. The time needed to clear the passivation is very short in standard TMAP processes. However, this effect is not negligible for nano-scale etching, as the passivation etching time becomes comparable with the total $\mathrm{SF}_{6}$ time step. Similarly, thicker passivation films reduce the $\mathrm{Si}$ etching rate [16] since a larger fraction of the $\mathrm{SF}_{6}$ step is needed to remove the passivation. To keep the etching rate low, the time step and flow rate of $\mathrm{SF}_{6}$ needs to be kept as low as possible. Thin passivation layers should be used in order to avoid micrograss formation [16]. The downside of very thin passivation layers is that ion induced defluorination, the ion bombardment assisted desorption of fluoride from the carbofluride film, creates chemical species in the passivation film that etches away the underlying $\mathrm{Si}[19,20]$. The favorable aspect of low $\mathrm{SF}_{6}$ flow rate is reduced roughness due to higher local plasma uniformity [18]. This effect can be exploited to affect the cross-section of the nanowires with the following mechanism: (1) The diameter of the nanowire is reduced isotropically (2) Sharp points and low dimension features are removed first, creating an elliptical shape.

\section{Results and discussion}

The etching of vertically stacked SiNW arrays is investigated for the following designs:

- Straight-line nanowire stacks.

- Curved-line nanowire stacks.

- Ultra-high density arrays.

Process development has been carried out by parameterizing individual device design in order achieve a high repeatability and fabrication yield. In Table 2 the design parameters are listed. In the following sections each device type is addressed separately. 
Table 1

Experimental design and process parameter dependencies with the main physical phenomena affecting the nano-scale TMAP process.

\begin{tabular}{|c|c|c|c|c|}
\hline Phenomena & Design variation & Process variation & Common problems & Effect \\
\hline Loading [17] & $\begin{array}{l}\text { Reduce exposed } \\
\text { area }\end{array}$ & $\mathrm{N} / \mathrm{A}$ & $\mathrm{N} / \mathrm{A}$ & Reduced etching rate \\
\hline $\begin{array}{l}\text { Microloading } \\
{[17,18]}\end{array}$ & Denser patterns & $\begin{array}{l}\text { Reduce } \mathrm{SF}_{6}(1) \text { Flow rate, }(2) \\
\text { Time step }\end{array}$ & $\begin{array}{l}\text { (1) Micrograss [16], (2) Formation of fins } \\
\text { instead of SiNWs }\end{array}$ & $\begin{array}{l}\text { (1) Reduced etching rate, (2) Reduced } \\
\text { roughness [18] }\end{array}$ \\
\hline IIDPF $[19,20]$ & $\mathrm{N} / \mathrm{A}$ & $\begin{array}{l}\text { Reduce } \mathrm{C}_{4} \mathrm{~F}_{8}(1) \text { Flow rate, }(2) \\
\text { Time step }\end{array}$ & Complete etched away structures & $\begin{array}{l}\text { Alter cross section from rhombic to } \\
\text { elliptical }\end{array}$ \\
\hline
\end{tabular}

Table 2

Mask design parameters for straight lines, curved lines and dense array nanowire structures.

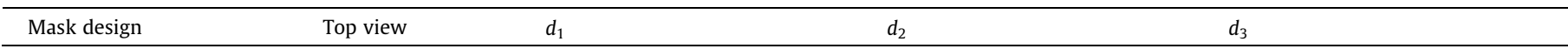

Straight-line nanowire stacks

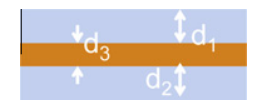

Trench opening: $100,200,500 \mathrm{~nm}$
Nanowire width: $50,100,150 \mathrm{~nm}$

Nanowire width: $50 \mathrm{~nm}$

Outer diameter: $250,450,850 \mathrm{~nm}$
Curved-line nanowire stacks

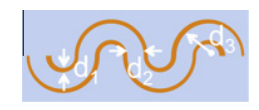

Trench opening: $200 \mathrm{~nm}$
Nanowire width: $50 \mathrm{~nm}$

(2)

Ultra-high density arrays

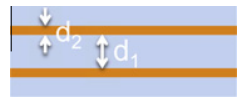

Pitch: 50, 60, 75, 100, $200 \mathrm{~nm}$
Nanowire width: $50 \mathrm{~nm}$

N/A

Table 3

Recipes overview optimized for different mask designs. The substrate temperature is kept at $20^{\circ} \mathrm{C}$.

\begin{tabular}{|c|c|c|c|c|c|}
\hline Process recipes & $\mathrm{SF}_{6}\left(\mathrm{~cm}^{3} / \mathrm{min}\right)$ & $\mathrm{SF}_{6}(\mathrm{~s})$ & $\mathrm{C}_{4} \mathrm{~F}_{8}\left(\mathrm{~cm}^{3} / \mathrm{min}\right)$ & $\mathrm{C}_{4} \mathrm{~F}_{8}(\mathrm{~s})$ & Mask design (see Table 2) \\
\hline (1) & 300 & 3 & 150 & 1 & Straight-line nanowire stacks \\
\hline$(2)$ & 200 & 2.5 & 150 & 1 & Curved-line nanowire stacks, ultra-dense arrays $\left(d_{1}=75,100,200 \mathrm{~nm}\right)$ \\
\hline (3) & 200 & 2.5 & 100 & 1 & Ultra-dense arrays $\left(d_{1}=50,60,75 \mathrm{~nm}\right)$ \\
\hline
\end{tabular}

\subsection{Straight-line nanowire stacks}

Lines widths of $50 \mathrm{~nm}, 100 \mathrm{~nm}, 150 \mathrm{~nm}$ are written with lengths $100 \mathrm{~nm}, 200 \mathrm{~nm}, 0.5 \mu \mathrm{m}, 1 \mu \mathrm{m}, 2 \mu \mathrm{m}$. The patterns are placed inside a trench to reduce and control the etching rate through microloading. Trench opening of $100 \mathrm{~nm}, 200 \mathrm{~nm}$ and $500 \mathrm{~nm}$ are designed. Flow rate used are $300 \mathrm{~cm}^{3} / \mathrm{min}$ for $\mathrm{SF}_{6}$ and $150 \mathrm{~cm}^{3} / \mathrm{min}$ for $\mathrm{C}_{4} \mathrm{~F}_{8}$ with step times have $3 \mathrm{~s}$ and $1 \mathrm{~s}$, respectively. The process can achieve very good repeatability, with a wire diameter variation of less than $10 \mathrm{~nm}$. The smallest fabricated structures for the straight-line design are nanowires obtained from $50 \mathrm{~nm}$ mask line width, $200 \mathrm{~nm}$ trench opening and of independent length. In Fig. 2a and b a stack of six nanowires is shown in bird's eye view and cross-section, respectively. The importance of the trench opening dimension is established by the observation that, for $500 \mathrm{~nm}$ trench opening nanowires are not formed (see Fig. 2c) while for $200 \mathrm{~nm}$ trench opening, while using the same process, $50 \mathrm{~nm}$ nanowires are fabricated (as shown in Fig. 2b).
The trench opening etching rate dependence can be explained by the microloading effect causing local etchant depletion.

\subsection{Curved-line nanowire stacks}

Curved line nanowires are patterned with a line width of $50 \mathrm{~nm}$. The patterns are carved into $\mathrm{Si}$ as for the case of straight-line nanowires, as depicted in Fig. 3a. The trench opening is kept constant at $200 \mathrm{~nm}$. The pattern consists of semi-circles connected in a serpentine fashion. Three circle diameters are explored: $200 \mathrm{~nm}, 400 \mathrm{~nm}$, and $800 \mathrm{~nm}$, as shown in Fig. 3c. In Fig. 3b a bird's eye view of a stack of three nanowires with $200 \mathrm{~nm}$ circle diameter encased in Pt is shown.

A drawback of the design is that the trench opening cannot be kept constant in case the circle diameter is double the trench opening, as can been seen from Fig. 3c by comparing the designs with $200 \mathrm{~nm}$ and $800 \mathrm{~nm}$ diameter. Etching rate is faster where trench opening is wider, again due to microloading effect. To reduce the 


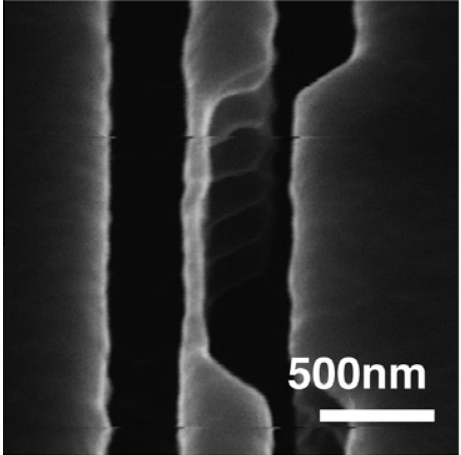

a

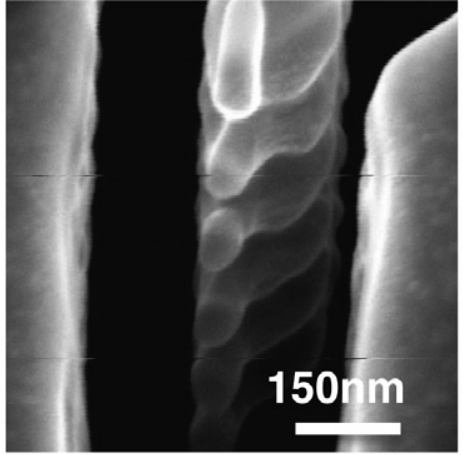

b

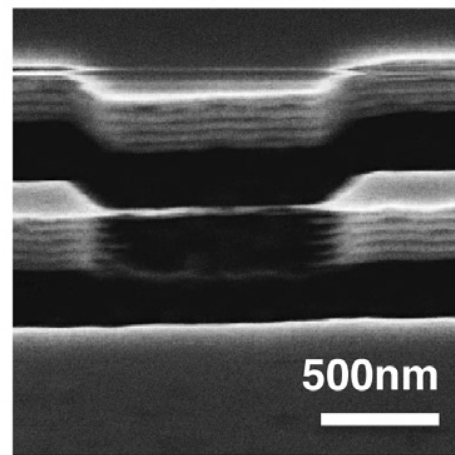

C

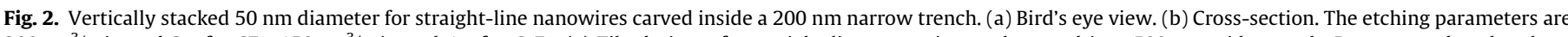

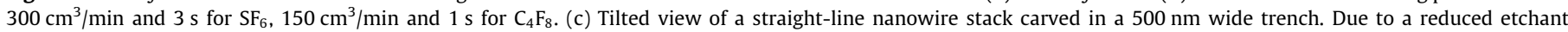
depletion effect, all the nanowires but the top one are overetched.

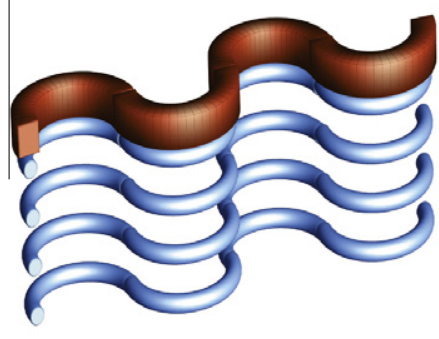

a

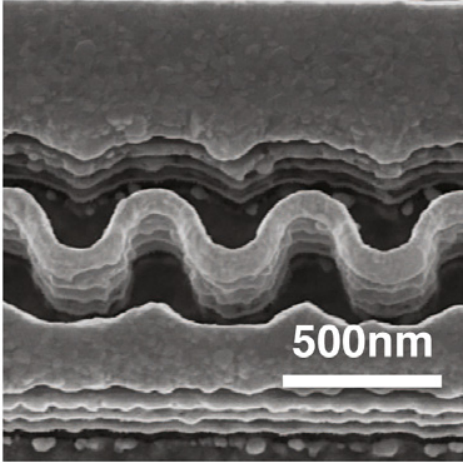

b

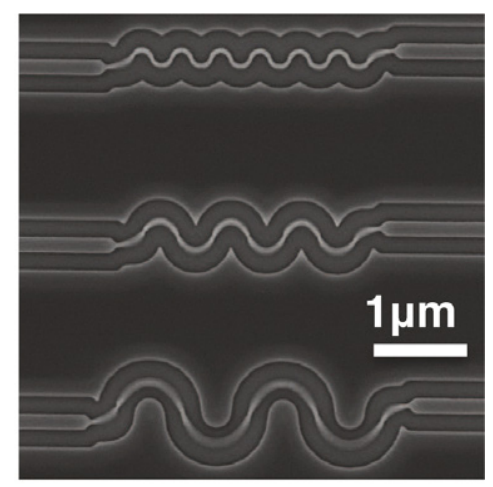

C

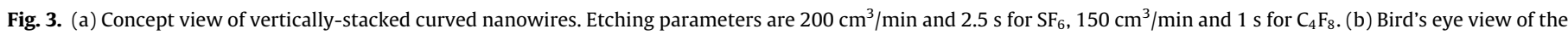
nanowire stack covered by a $50 \mathrm{~nm}$ thick Pt layer for conformal PtSi silicide formation. (c) Top view of the three curved nanowire design.

etching rate created by the irregular trench openings a reduced flow rate recipe is used. Flow rate of $200 \mathrm{~cm}^{3} / \mathrm{min}$ for $\mathrm{SF}_{6}$ and $150 \mathrm{~cm}^{3} /$ min for $\mathrm{C}_{4} \mathrm{~F}_{8}$ were used where step times are $2.5 \mathrm{~s}$ and $1 \mathrm{~s}$, respectively.

\subsection{Ultra-high density arrays}

Dense array designs for $50 \mathrm{~nm}$ line widths and pitches ranging from $100 \mathrm{~nm}$ to $300 \mathrm{~nm}$ were explored for lengths from $100 \mathrm{~nm}$ to $50 \mu \mathrm{m}$. The array pattern was etched faster than the reference case of the straight line nanowires explained in Section 4.1. This could be due to the vertical nanowire separation that alters the etchants flow once the nanowire is released as illustrated in Fig. 4, countering this effect is microloading that slows down etching rate the denser the array gets. Two recipes are selected: the first is optimized for $200 \mathrm{~nm}$ trench opening and it uses $200 \mathrm{~cm}^{3} / \mathrm{min}$ for $\mathrm{SF}_{6}$ in $2.5 \mathrm{~s}$ time steps and $150 \mathrm{~cm}^{3} / \mathrm{min}$ for $\mathrm{C}_{4} \mathrm{~F}_{8}$ in $1 \mathrm{~s}$ time steps. In Fig. 5 a stack of 3 nanowires, which is fabricated with the first recipe, is shown in bird's eye view and cross-section. Average diameter of the SiNWs is $30 \mathrm{~nm}$ and pitch is $200 \mathrm{~nm}$. The length of the nanowires is $1 \mu \mathrm{m}$. The same recipe is used to fabricate a stack of six round SiNWs with pitch of $75 \mathrm{~nm}$ and $300 \mathrm{~nm}$ long (Fig. 6a) and SiNW diameters of $20 \mathrm{~nm}$ (Fig. 6b), producing repeatable SiNW structures with minimal diameter variation. The second recipe is designed for denser arrays and uses $200 \mathrm{~cm}^{3} / \mathrm{min}$ for $\mathrm{SF}_{6}$ for $2.5 \mathrm{~s}$ and $100 \mathrm{~cm}^{3} / \mathrm{min}$ for $\mathrm{C}_{4} \mathrm{~F}_{8}$ for $1 \mathrm{~s}$. A stack of six rhombic SiNWs with pitch of $75 \mathrm{~nm}$ (Fig. 7a) is fabri-

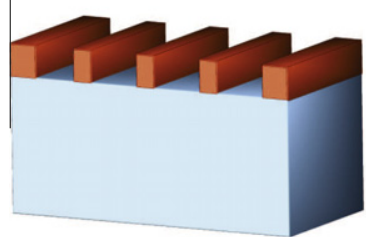

a

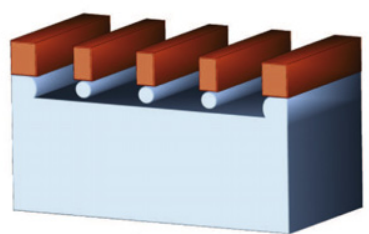

b

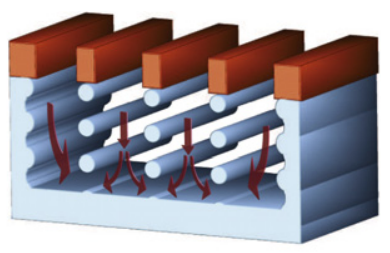

C

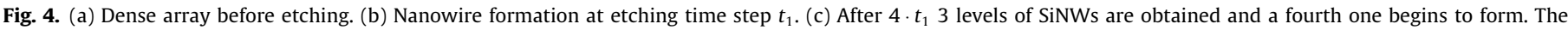
arrows indicate the plasma flow directions. 


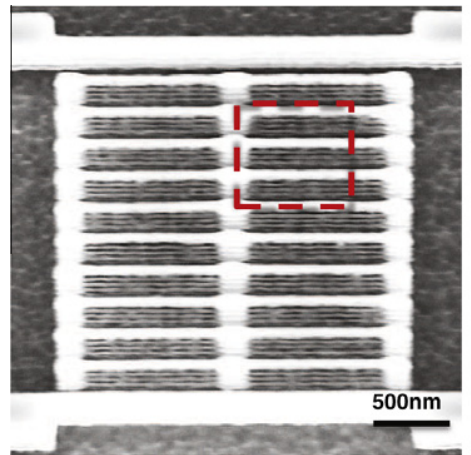

a

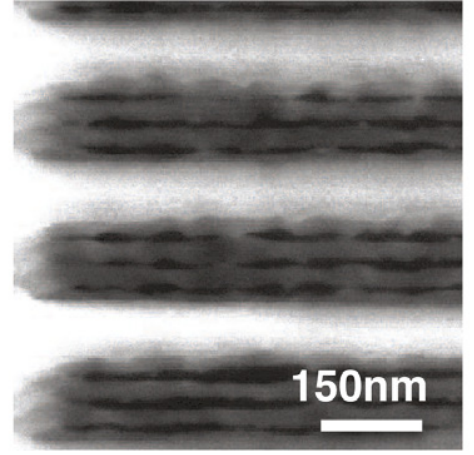

b

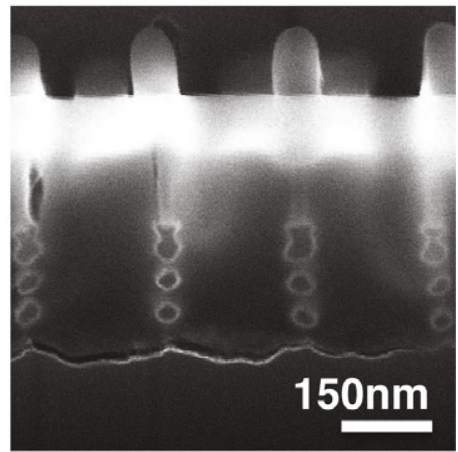

C

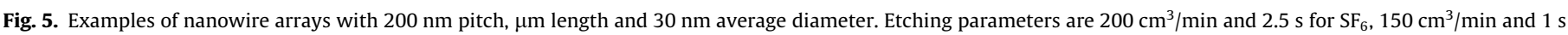
for $\mathrm{C}_{4} \mathrm{~F}_{8}$. (a) Bird's eye view. (b) High magnification showing the vertical stacks. (c) Cross-section of the vertically stacked array.

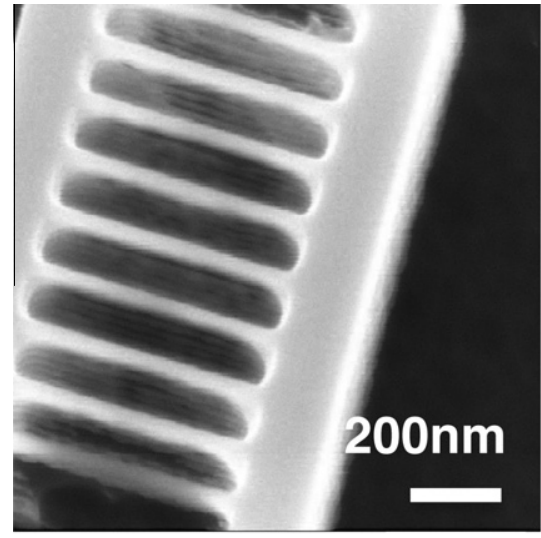

a

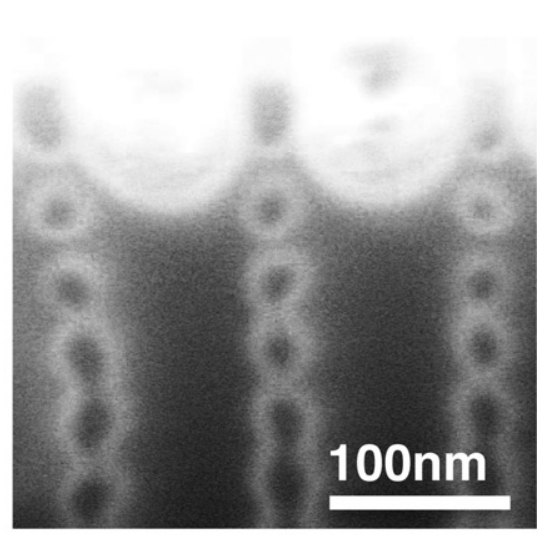

b

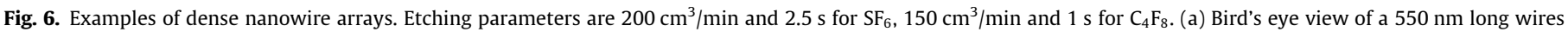
with $200 \mathrm{~nm}$ pitch cut with FIB. (b) Cross-section for an array with $300 \mathrm{~nm}$ long wires and $100 \mathrm{~nm}$ pitch. The average nanowire diameter is $25 \mathrm{~nm}$.

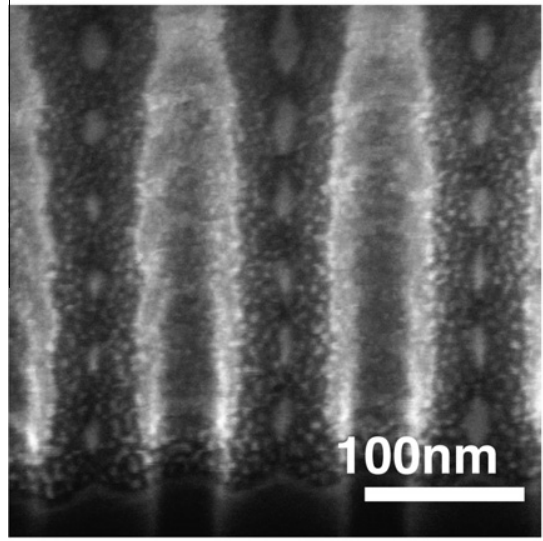

a

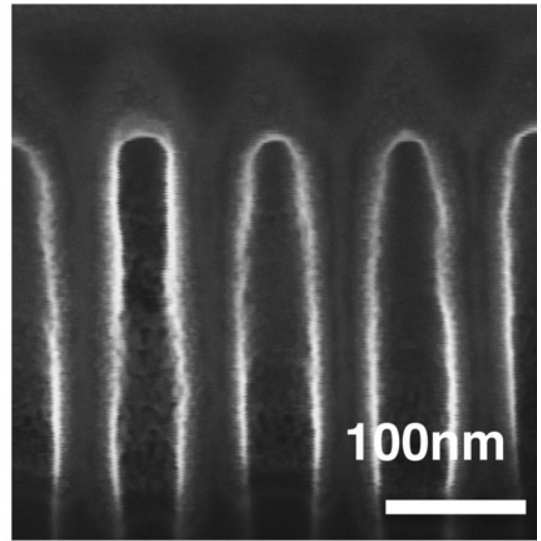

b

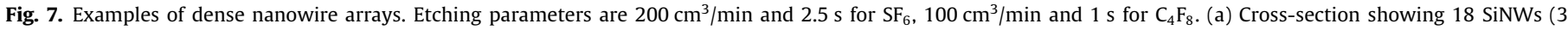

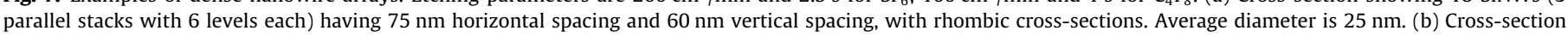
showing scalloped fins for $50 \mathrm{~nm}$ horizontal spacing (dimension $\mathrm{d}_{1}$ for dense array design as in Table 2).

cated for $75 \mathrm{~nm}$ horizontal and $60 \mathrm{~nm}$ vertical spacings. Typical SiNW diameter is $10 \mathrm{~nm}$ with lengths of up to $550 \mathrm{~nm}$, yet diameter variability is larger compared to earlier examples. Higher density arrays lead to the formation of scalloped fins, as shown in Fig. 7b.

\section{Conclusions}

Vertical stacks of SiNWs with round or rhombic shapes of diameters down to $10 \mathrm{~nm}$ are fabricated using a simple TMAP process in 
bulk $\mathrm{Si}$ with very-low thermal budget. To our knowledge, the cross-sectional nanowire density of $2 \times 10^{10} / \mathrm{cm}^{2}$ result is among the highest for top-down fabricated SiNWs arrays reported in the literature. Moreover, SiNWs arrays with lengths from $50 \mathrm{~nm}$ up to $1 \mu \mathrm{m}$ and horizontal inter-wire spacing ranging between $50 \mathrm{~nm}$ and $200 \mathrm{~nm}$ are demonstrated. The technique is presented in terms of its potential use for the fabrication of a broad range of nano-scale devices, from NEMS, to nano-electronics and sensing.

\section{Acknowledgments}

The authors would like to acknowledge the CMI staff of EPFL for help with the fabrication. This work has been partially supported by the Swiss NSF Grant No. 200021-122168, Swiss NSF Grant No. 200021-132539.

\section{References}

[1] K.L. Ekinci, M.L. Roukes, Review of Scientific Instruments 76 (6) (2005) 061101 (pages 12).

[2] J. Hu, T.W. Odom, C.M. Lieber, Accounts of Chemical Research 32 (5) (1999) 435-445.

[3] F. Patolsky, G. Zheng, C.M. Lieber, Natural Protocols 1 (4) (2006) 1711-1724.

[4] Y. Cui, C.M. Lieber, Science 291 (5505) (2001) 851-853.

[5] N.A. Melosh, A. Boukai, F. Diana, B. Gerardot, A. Badolato, P.M. Petroff, et al., Science 300 (5616) (2003) 112-115.

[6] A. Javey, Nam, R.S. Friedman, H. Yan, C.M. Lieber, Nano Letters 7(3) (2007) 773-777.
[7] T. Ernst, C. Dupre, C. Isheden, E. Bernard, R. Ritzenthaler, V. Maffini-Alvaro et al., Novel 3D integration process for highly scalable nano-beam stackedchannels GAA (NBG) FinFETs with HfO2/TiN gate stack, in: Electron Devices Meeting, 2006, IEDM '06, International, 2006, p. 1-4.

[8] N. Singh, K. Buddharaju, S. Manhas, A. Agarwal, S. Rustagi, G. Lo, et al., IEEE Transactions on Electron Devices 55 (11) (2008) 3107-3118.

[9] P. Hashemi, M. Canonico, J.K. Yang, L. Gomez, K.K. Berggren, J. Hoyt, ECS Transactions 16 (2008) 57-68.

[10] G.F. Cerofolini, M. Ferri, E. Romano, F. Suriano, G.P. Veronese, S. Solmi, et al., Semiconductor Science Technology 25 (9) (2010) 095011. -+.

[11] M. Ferri, F. Suriano, A. Roncaglia, S. Solmi, G. Cerofolini, E. Romano, et al., Microelectronic Engineering 88 (6) (2011) 877-881.

[12] O. Ozsun, B. Alaca, Y. Leblebici, A. Yalcinkaya, I. Yildiz, M. Yilmaz, et al., Journal of Microelectromechanical Systems 18 (6) (2009) 1335-1344.

[13] D. Sacchetto, M. Ben-Jamaa, G. De Micheli, Y. Leblebici, Fabrication and characterization of vertically stacked gate-all-around Si nanowire FET arrays, in: Solid State Device Research Conference, 2009, ESSDERC '09, Proceedings of the European, 2009, p. 245-248.

[14] R. Ng, T. Wang, F. Liu, X. Zuo, J. He, M. Chan, IEEE Electron Device Letters 30 (5) (2009) 520-522.

[15] H.C. Yuan, Z. Ma, Applied Physics Letters 89 (21) (2006) 212105 (pages 3).

[16] B. Wu, A. Kumar, S. Pamarthy, Journal of Applied Physics 108 (5) (2010) 051101 (pages 20).

[17] C. Hedlund, H.O. Blom, S. Berg, Journal of Vacuum Science Technology A: Vacuum, Surfaces, and Films 12 (4) (1994) 1962-1965.

[18] R.A. Gottscho, C.W. Jurgensen, D.J. Vitkavage, Journal of Vacuum Science Technology B: Microelectronics and Nanometer Structures 10 (5) (1992) 2133-2147.

[19] T.E.F.M. Standaert, C. Hedlund, E.A. Joseph, G.S. Oehrlein, T.J. Dalton, Journal of Vacuum Science and Technology A: Vacuum, Surfaces, and Films 22 (1) (2004) 53-60.

[20] T.E.F.M. Standaert, M. Schaepkens, N.R. Rueger, P.G.M. Sebel, G.S. Oehrlein, J.M Cook, International Workshop on Basic Aspects of Nonequilibrium Plasmas Interacting with Surfaces (BANPIS97) 16 (1) (1998) 239-249. 